Technological University Dublin

DƯBLIN

ARROW@TU Dublin

2008-01-01

\title{
Fabrication of Switchable Liquid Crystal Devices using Surface Relief Gratings in Photopolymer
}

\author{
Pavani Kotakonda \\ Technological University Dublin \\ Izabela Naydenova \\ Technological University of Dublin, izabela.naydenova@tudublin.ie \\ Robert Howard \\ Technological University of Dublin, Robert.Howard@tudublin.ie
}

See next page for additional authors

Follow this and additional works at: https://arrow.tudublin.ie/cieoart

Part of the Condensed Matter Physics Commons, and the Optics Commons

\section{Recommended Citation}

Kotakonda, P. et al. (2008) Fabrication of switchable liquid crystal devices using surface relief gratings in photopolymer. Journal of Material Science (materials in electronics) (JMSE) doi:10.1007/

s10854-007-9537-5

This Article is brought to you for free and open access by the Centre for Industrial and Engineering Optics at ARROW@TU Dublin. It has been accepted for inclusion in Articles by an authorized administrator of ARROW@TU Dublin. For more information, please contact arrow.admin@tudublin.ie, aisling.coyne@tudublin.ie, gerard.connolly@tudublin.ie.

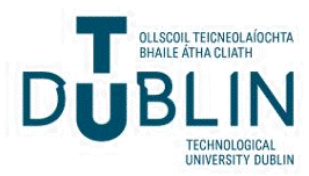




\section{Authors}

Pavani Kotakonda, Izabela Naydenova, Robert Howard, Suzanne Martin, and Vincent Toal 


\title{
Fabrication of switchable liquid crystal devices using surface relief gratings in photopolymer
}

\author{
K. Pavani ${ }^{1,2}$, I. Naydenova ${ }^{1}$, R. Howard ${ }^{2}$, S. Martin ${ }^{1}$ and V. Toal ${ }^{1,2}$ \\ ${ }^{1}$ Centre for Industrial and Engineering Optics, ${ }^{2}$ School of Physics \\ Dublin Institute of Technology, Kevin Street, Dublin 8, Ireland.
}

\begin{abstract}
Holographically recorded surface relief gratings in dry, self-developing acrylamide based photopolymer were used to fabricate two types of switchable liquid crystal (LC) device. One is an electrically switchable LC diffraction grating and the other is an electrically switchable polarization rotator. The electrically switchable diffraction grating was characterized by measuring the dependence of the intensity in the first diffracted order on the applied electric field. The polarization rotator was characterized by studying the influence of the applied electric field on the twist angle and the variation of intensity in the zero and the first orders of diffraction.
\end{abstract}

\section{Introduction}

The applications of switchable holographic optical devices are in beam steering, light intensity modulation (displays) and in optical communications. Switchable optical devices generally use materials that exhibit a change in their refractive index when an electrical field is applied. Liquid crystals (LCs) can exhibit large changes in refractive index [1, 2] arising from the reorientation of their, usually, rod-shaped polar molecules under the influence of an electric field. There are several methods for fabricating these switchable LC devices [2]. Of which optical recording of surface relief gratings in photopolymer is one attractive method [2-6]. An acrylamide based photopolymer developed at the Centre for Industrial and Engineering Optics was used in this work so as to expand the capability of this material for different applications [7-10]. The material consists of monomers, an electron donor, a photosensitizer and a polymer binder. Optical recording in this material is based on the photoinduced polymerization process [11]. The photopolymer layer is exposed to an interference pattern and the photosensitizer absorbs a photon, triggering the polymerisation process in the bright regions which results in several reaction processes. Due to these reactions, a change in the refractive index or a change in the thickness of the material occurs. The variation of thickness as a result of photopolymerization is referred as a surface relief grating. Previous studies on photoinduced surface relief gratings in this material $[11,12]$ strongly indicate that a concentration gradient created by nonuniform polymerization causes mass transport of monomer from dark to bright regions and this is the main mechanism which governs the formation of surface relief gratings. Extensive investigation of the dependence of the photoinduced surface relief gratings on physical and recording parameters was reported earlier $[11,12]$. Switchable LC diffraction gratings were successfully fabricated by filling the surface relief gratings of spatial frequency 70 lines/mm with E49 LCs and the switching behaviour was demonstrated [13]. In this paper, we report a comparison between the behaviour of the switchable diffraction gratings using E7 $\left(\mathrm{n}_{\mathrm{e}}=1.74, \mathrm{n}_{\mathrm{o}}=\right.$ 1.52) and E49 $\left(\mathrm{n}_{\mathrm{e}}=1.79, \mathrm{n}_{\mathrm{o}}=1.53\right)$ LCs from Merck and the switching behaviour was demonstrated in each case. Polarization rotation twisted nematic LC devices were also fabricated with ZLI3700-000 $\left(\mathrm{n}_{\mathrm{e}}=1.58, \mathrm{n}_{\mathrm{o}}=1.48\right)$ LCs also from Merck by using the surface relief effect along with rubbing to align the LCs. The device consists of two photopolymer layers with sinusoidal surface relief profile obtained by holographic recording. The device is assembled in such a way that the 
wave vectors of the two gratings are orthogonal. Before assembly, the surface relief gratings were rubbed five times along the fringes with a velvet cloth. The assembled device was then filled with the LCs. The main difference between the device presented here and the devices fabricated by the standard rubbing technique is that, in addition to exhibiting electro-optical polarisation rotation behaviour, it also acts as a switchable beam splitter due to the existence of the diffraction orders. The twisted nematic liquid crystal device was placed between crossed polarizers and the influence of the applied electric field on the twist angle and on the intensities in the zero and first orders of diffraction was studied.

\section{Experimental details}

The sample preparation and the optical setup for the fabrication of the surface relief gratings are described elsewhere [11]. The fabrication of LC diffraction gratings and the optical setup to study their electro-optical switching behaviour has also been described [13]. A laser beam, wavelength $532 \mathrm{~nm}$, was spatially filtered and collimated to record surface relief transmission gratings of spatial frequency 100 lines $\mathrm{mm}^{-1}$. A vertically polarised laser beam of wavelength $633 \mathrm{~nm}$ was used to characterise the devices using the experimental set up shown in fig 1. A half wave plate is used to ensure that the plane of polarization of the incident light is parallel to the input director of the device and an analyzer is set with its transmission axis orthogonal to the plane of polarization of the input light beam.

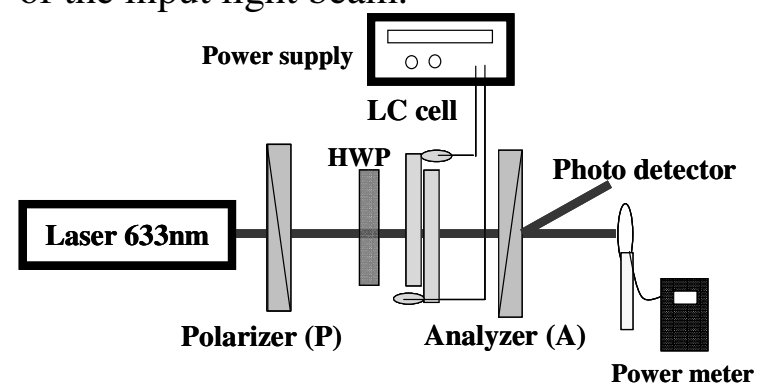

Fig. 1 Optical setup used to characterize the twisted nematic LC device
The director in the twisted nematic LC device is determined as follows. Suppose the input plane of polarization makes a small, unknown angle $\delta$, with the director at the input of the device. If the amplitude of the electric field at the input is A then the components parallel and perpendicular to the director are $A \cos \delta$ and $A \sin \delta$. Both components will be rotated by $\alpha$, the twist angle.

Now suppose an analyzer is inserted at the output, with its transmission axis set at an unknown angle $\gamma$ to the output director of the device. The output intensity is given by

$$
\begin{aligned}
& \mathrm{I}=(\mathrm{A} \cos \delta \cos \gamma)^{2}+(\mathrm{A} \sin \delta \sin \gamma)^{2} \\
& =\mathrm{A}^{2}\left[\left(1-\sin ^{2} \delta\right) \cos ^{2} \gamma+\sin ^{2} \delta \sin ^{2} \gamma\right] \\
& =\mathrm{A}^{2}\left[\cos ^{2} \gamma+\sin ^{2} \delta\left(\sin ^{2} \gamma-\cos ^{2} \gamma\right)\right]
\end{aligned}
$$

When $\gamma=0, I=A^{2} \cos ^{2} \delta$ (maximum) and when $\gamma=\pi / 2, I=A^{2} \sin ^{2} \delta$ (minimum).

It follows that when the output is maximum, the analyzer has its transmission axis parallel to the output director.

Further, when $\delta$ is zero, the intensity is $\mathrm{A}^{2}$, so the half wave plate in front of the input director should be rotated about the optical axis to obtain an absolute maximum output. The input plane of polarization is now parallel to the input director. The twist angle is obtained from the settings of the half wave plate and the analyzer. It is the angle between the input light polarization direction and transmission axis of the analyzer.

The basic operation of the twisted nematic LC device as a polarization rotator is shown in fig 2 and is explained as follows. When no electric field is applied to the device (OFF state), the linearly polarized light propagates through the LC layer and its plane of polarization is rotated by the twisted structure and the light emerges from the layer with its polarization plane parallel to the transmission axis of the analyzer. When a voltage is applied across 
the device (ON state), the director (local optic axis) in the central portion of the LC layer orients parallel to the electric field and the twist is removed. The polarization plane of the light is no longer rotated and light passing through the cell is absorbed at the analyzer which is in the crossed position $[1,2]$.

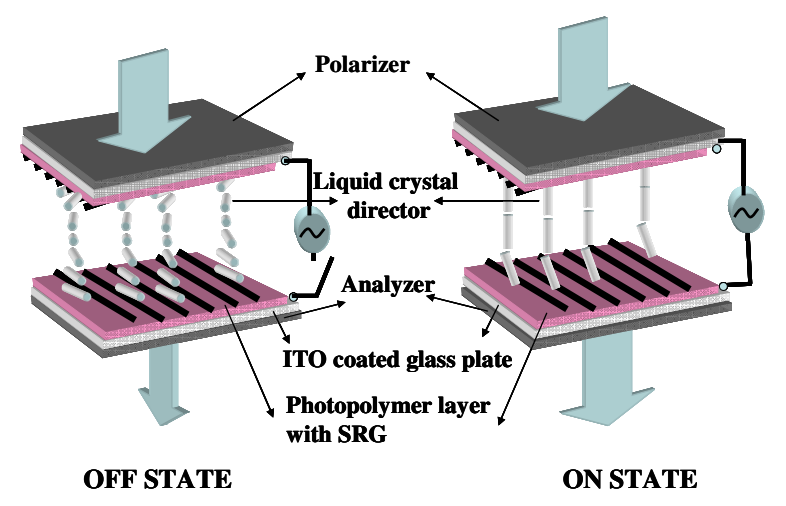

Fig 2 Operation of the twisted nematic LC device

\section{Results and discussions}

\section{Switchable LC diffraction gratings}

The surface relief gratings were recorded in a $10 \mu \mathrm{m}$ thick photopolymer layer deposited on an ITO coated glass plate. The recording intensity was $10 \mathrm{~mW} / \mathrm{cm}^{2}$ and exposure time was $35 \mathrm{~s}$. The gratings were post-exposed to a uniform UV light source of $16 \mathrm{~W}$ for $20 \mathrm{~min}$. The surface relief amplitude modulation of the grating was measured with a white light surface profilometer and a value of $129 \pm 8 \mathrm{~nm}$ was obtained. The diffraction efficiency (DE) of the gratings was $7 \pm 0.5 \%$ before filling with LCs. The gratings were filled with E49 or E7 LCs. The DE after filling was measured to be $0.4 \%$ for E49 LCs and $0.15 \%$ for E7 LCs. The decrease in the DE could be explained by the fact the refractive index difference between polymer and LCs is smaller than the refractive index difference between polymer and air [13]. The DE values for E7 LC gratings were less than those for E49 LC gratings which could be explained by a higher difference between the average refractive index of E49 LCs and that of the photopolymer.

Fig 3 shows the switching behaviour of the LC diffraction gratings. Both types show similar switching behaviour, switching OFF at around $1.5 \mathrm{~V}$ and $1 \mathrm{~V}$, when LCs must have realigned such that the refractive index of photopolymer matched that of the LCs. The switching time from $\mathrm{ON}$ to OFF state was around $10 \mathrm{~s}$ and 60-70 s from OFF to ON state.

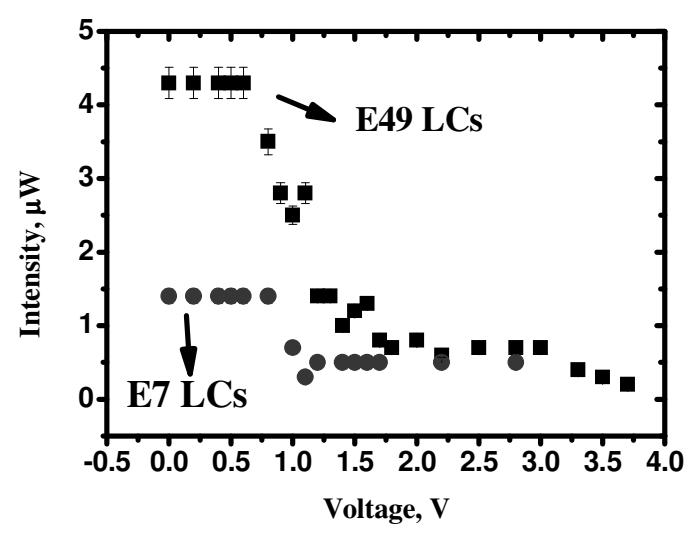

Fig 3 Switching behaviour of the LC diffraction gratings

\section{Twisted nematic LC device}

The twisted nematic LC device was fabricated by sandwiching ZLI-3700-000 LCs between rubbed surface relief gratings separated by $6 \mu \mathrm{m}$ spacers and placed with their grating vectors at right angles to each other. Fig 4 shows the variation of the normalised transmitted intensity with applied electric field when the device is placed between crossed polarizers. Fig 5 shows the variation in the twist angle with applied voltage. It is seen that at around $3.4 \mathrm{~V}$ the twist disappears probably due to orientation of liquid crystals perpendicular to the substrates. The polarization plane of the light passing through the device is not rotated and the light is then absorbed at the analyzer as shown in fig 4 . The time taken to switch from the OFF state to ON was 6-8 $\mathrm{s}$ and $12-15 \mathrm{~s}$ from the $\mathrm{ON}$ to the OFF state. 


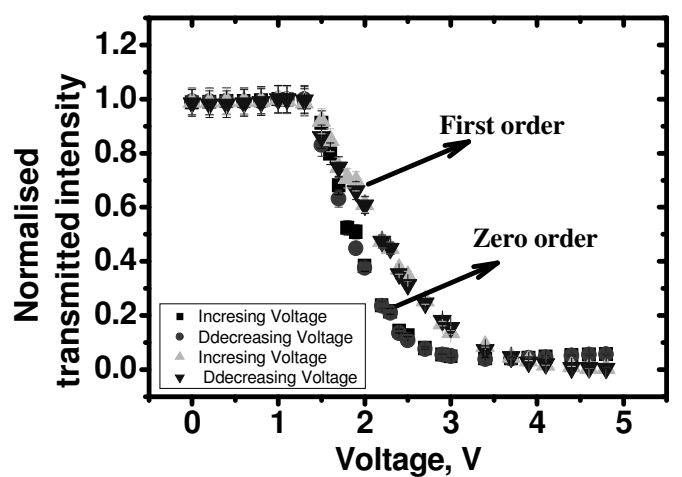

Fig 4 Intensity verses voltage

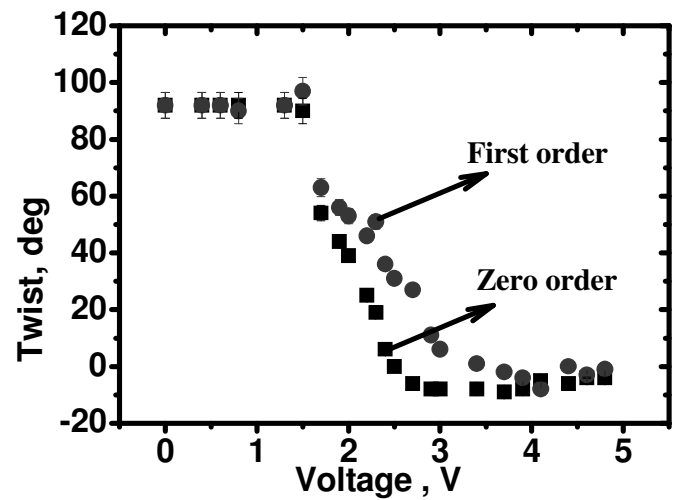

Fig 5 Twist angle verses voltage

From these results it is observed that when no electric field is applied a twist of $92^{\circ}$ is obtained, and it can be decreased with applied voltage but the original twist angle is restored when the voltage is removed. The same twist angle and similar switching behaviour was also observed in the first diffracted order. The analysis of the polarization state of the light emerging from the device is in progress.

\section{Conclusions}

The potential of optically recorded surface relief gratings in IEO photopolymer for fabricating LC devices has been demonstrated. LC diffraction gratings were fabricated with two different LCs. The gratings prepared with higher $\mathrm{n}_{\mathrm{e}}$ LCs show higher DE. Photoinduced surface relief gratings in combination with rubbing were used to orient liquid crystals for the successful fabrication of a twisted nematic liquid crystal device for rotating the plane of polarization of light. To the best of our knowledge an acrylamide-based photopolymer is used for the first time to fabricate this type of device. A twist angle of $92^{\circ}$ was observed in the zero and first orders. The effects of an applied electric field on the transmitted and the first order diffracted light intensities and on the twist angle were studied.

\section{Acknowledgements}

K. Pavani would like to acknowledge Dublin Institute of Technology, Faculty of Science for financial support.

\section{References}

1. B. Bahadur, vol.1 World Scientific, (1990).

2. K. Takatoh, M. Hasegawa, M. Koden, N. Itoh, R. Hasegawa, M. Sakamoto, Taylor \& Francis (2005).

3. X. T. Li, A. Natansohn, P. Rochon, Appl. Phys. Lett., 74 (25) ( 1999).

4. X. T. Li , A. Natansohn, S. Kobayashi and P. Rochon,, IEEE J. Quantum. Electronics., 824827, 36(7) (2000)

5. D. Dantsker, J. Kumar, S. K. Tripathy, J.Appl. Phys, 4318-4325, 89 (8) (2001).

6. M.O Neill and S.M.Kelly, J.Phys.D.Appl.Phys, 67-84 (33) (2000).

7. S. Martin, C. A. Feely and V. Toal, Appl. Opt., 5757-5768, 36 (1997).

8. R. Jallapuram, I. Naydenova, S. Martin, R. Howard, V. Toal, Sven Frohmann, Susanna Orlic, Hans J Eichler, Opt. Mater, 1329-1333, 28 (2006).

9. S.Guntaka, V.Toal, S.Martin, Appl.Opt. 74757479, 41(35) (2002).

10. H. Sherif, I. Naydenova, S. Martin, C. Mc Ginn, V. Toal, J. J. Opt .A: Pure Appl. Opt., 255-260, 7(2005).

11. K. Pavani, I. Naydenova, S. Martin, V. Toal, J.Opt.A: Pure Appl. Opt., 43-48, 9 (2007).

12. I.Naydenova, E.Mihaylova, S.Martin, V.Toal, Opt. Express, 4878-488, 13(13) (2005).

13. K. Pavani, I. Naydenova, S. Martin, R. Jallapuram, R. G. Howard, V. Toal, Opt. Commun., 367-369 273(2) (2007). 